\title{
Subclinical Vascular Disease Burden and Premature Mortality Among Middle-aged Adults: the Atherosclerosis Risk in Communities Study
}

\author{
Chenkai Wu, Ph.D., M.P.H., M.S. ${ }^{1,2}{ }^{\mathbb{1}}$, Kehan Zhang, B.S. ${ }^{7}$, Michelle C. Odden, Ph.D. ${ }^{3}$, \\ Anna M. Kucharska-Newton, Ph.D.4, Priya Palta, Ph.D. ${ }^{4,5}$, Kunihiro Matsushita, M.D.6, \\ Rebecca F. Gottesman, M.D., Ph.D. ${ }^{7,8}$, and B. Gwen Windham, M.D. ${ }^{9}$
}

\begin{abstract}
'Global Health Research Center, Duke Kunshan University, Kunshan, Jiangsu, China; '2Duke Global Health Institute, Duke University, Durham, NC, USA; ${ }^{3}$ Department of Health Research and Policy, Stanford University, Stanford, CA, USA; ${ }^{4}$ Department of Epidemiology, University of North Carolina Gillings School of Global Public Health, Chapel Hill, NC, USA; ${ }^{5}$ Division of General Medicine, Department of Medicine, Columbia University., New York, NY, USA; ${ }^{6}$ Department of Epidemiology, Johns Hopkins Bloomberg School of Public Health, Baltimore, MD, USA; ${ }^{7}$ Department of Neurology, Johns Hopkins University School of Medicine, Baltimore, MD, USA; ${ }^{8}$ Department of Epidemiology, Johns Hopkins Bloomberg School of Public Health, Baltimore, MD, USA; ${ }^{9}$ Division of Geriatrics, Department of Medicine, University of Mississippi Medical Center, Jackson, MS, USA.
\end{abstract}

BACKGROUND: Whether high burden of subclinical vascular disease (SVD) is associated with increased premature mortality among middle-aged adults is not adequately understood. The association of midlife SVD burden with premature mortality among middle-aged adults free of clinical cardiovascular disease (CVD) could provide further insights into stratifying premature death beyond clinical CVD.

OBJECTIVE: To determine whether high burden of subclinical vascular disease is associated with increased premature mortality among middle-aged adults.

DESIGN: We leveraged data from the Atherosclerosis Risk in Communities Study.

PARTICIPANTS: Thirteen thousand eight hundred seventy-six community-dwelling blacks and whites aged 45-64 years from the Atherosclerosis Risk in Communities Study.

MAIN MEASURES: Each SVD measure-ankle-brachial index, carotid intima-media thickness, and electrocardiogram-was scored 0 (no abnormalities), 1 (minor abnormalities), or 2 (major abnormalities). An index was constructed as the sum of three measures, ranging from 0 (lowest burden) to 6 (highest burden). We used the Cox proportional-hazards model to determine the association of SVD burden with premature mortality (death before age 70) among persons free of clinical CVD. We then tested the difference in point estimates between SVD and clinical CVD.

KEY RESULTS: Among persons without CVD, the premature death was $1.7,2.1,2.5$, and 3.8 per 1000 personyears among those with an SVD score of 0 (lowest burden), 1, 2, and 3-6 (highest burden), respectively. After multivariable-adjustment, highest SVD burden (score = $3-6 ; \mathrm{HR}=1.47$ ) was significantly associated with premature death among persons initially without CVD. In the model where persons with and without CVD were

Supplementary Information The online version contains supplementary material available at https://doi.org/10.1007/s11606-020-06398-6.

Received June 28, 2020

Accepted December 3, 2020

Published online January 19, 2021 included, high SVD burden (score: 3-6 vs. 0) and CVD did not have hugely different association with premature death (HR $=1.49$ vs. $1.68 ; P=0.32$ for comparison).

CONCLUSIONS: Midlife SVD burden was associated with premature mortality and it could stratify premature death beyond clinical CVD. It is important to take SVD into account when designing interventions for reducing premature mortality.

KEY WORDS: atherosclerosis; cardiovascular diseases; vascular diseases; premature mortality.

J Gen Intern Med 36(7):2048-54

DOI: $10.1007 / \mathrm{s} 11606-020-06398-6$

(C) Society of General Internal Medicine 2021

\section{INTRODUCTION}

Reducing premature mortality, defined as death under age 70 , is an important issue of international concern. The Sustainable Development Goals (SDGs), which were adopted by the UN in 2015 , target a one-third reduction in premature mortality from 2010 to $2030 .^{1}$ Although there has been a tremendous increase in life expectancy over the last few centuries in the USA, a large number of American adults still die prematurely every year with most deaths attributable to non-communicable diseases (NCDs). The risk of dying from an NCD for a man and woman aged $30-70$ years was $18 \%$ and $12 \%$, respective$\mathrm{ly}^{2}$ Cardiovascular disease (CVD) is the leading cause of death both worldwide and nationwide, accounting for an estimated 17.9 million deaths each year globally and 1 in 3 deaths in the USA. ${ }^{3,4}$ Of all annual deaths caused by CVD globally, one-third of them have been identified as premature deaths. ${ }^{3}$ Each year, CVD causes 137.4 billion dollars in lost productivity from premature mortality in the USA. ${ }^{4}$

In addition to CVD, subclinical vascular disease (SVD) could also be an effective way to help recognize and prevent premature mortality. Noninvasive measures of SVD are known to be strong risk factors for cardiovascular events, 
frailty, and mortality among older adults, even after adjusting for traditional CVD risk factors. ${ }^{5-14}$ However, whether high burden of SVD is associated with increased mortality among middle-aged adults has not been adequately understood. We propose that middle adulthood (45-64 years of age) is a critical period of risk accumulation. Alternations to the cardiovascular system occurring in midlife may induce impairments across multiple organ systems, leading to increased mortality risk in older adulthood. Clinical CVD has a low prevalence in midlife $(<10 \%),{ }^{15}$ and therefore may not be able to fully account for vascular disease burden. In contrast, SVD varies substantially among asymptomatic persons and can track small alterations in structure and function of the cardiovascular system. Thus, measures of SVD may be useful to capture the full spectrum of cardiovascular comorbidity and could provide additional risk stratification of death beyond clinical CVD.

The purpose of the present study was two-fold. First, we examined the association between midlife SVD burden with premature mortality among middle-aged (45-64 years) adults who did not have clinical CVD. Second, we compared SVD burden and clinical CVD in terms of the association with premature mortality combining persons with and without CVD at baseline. We leveraged data from a large, longitudinal cohort of middle-aged, community-dwelling blacks and whites in the USA with over 25 years of follow-up-the Atherosclerosis Risk in Communities (ARIC) Study. Findings from this study might provide insights into the contribution of SVD to premature mortality among persons with and without clinically diagnosed CVD.

\section{METHODS}

\section{Data and Study Participants}

Data were from the Atherosclerosis Risk in Communities (ARIC) Study, an ongoing prospective cohort from four communities in the USA (suburbs of Minneapolis, Minnesota; Forsyth County, North Carolina; Washington County, Maryland, and Jackson, Mississippi). ${ }^{16}$ The ARIC Study sampled 15,792 men and women, aged 45-64 years at baseline (19871989), and was designed to examine the mechanisms of atherosclerosis and CVD risk factors. Extensive physical examinations were carried out at baseline and at three subsequent triennial clinical examinations: Visit 2 (1990-1992), visit 3 (1993-1995), and visit 4 (1996-1998). Ongoing follow-up of the ARIC cohort is conducted through annual telephone interviews, which have been semi-annual since 2012. Institutional review boards at each site approved the study protocol and all participants signed informed consent. More details about the recruitment strategies and study design of the ARIC Study can be found elsewhere. ${ }^{16}$

Participants were excluded from the present study if they (i) self-reported race other than black or white $(n=48)$, (ii) were blacks from the Minnesota and Maryland sites $(n=55$; a small sample size), (iii) had missing data on any of the three measures of SVD ( $n=1478)$, and (iv) were alive but not yet 70 at the cut-off date of December 31, $2015(n=332)$.

\section{Outcome}

The primary outcome was time to premature mortality (we used age as the time scale), defined as death before 70 years of age according to the definition in the UN SDGs. ${ }^{1}$ Participants who survived over the age 70 were censored. Mortality ascertainment was conducted using annual follow-up telephone calls and semi-annual calls since 2012 to participants or proxies, community-wide hospital surveillance, and linkage with local and national death registries. Mortality information was available through December 31, 2015.

\section{Components of the Subclinical Vascular Disease Burden}

Three measures of subclinical vascular health were assessed at baseline (1987-1989): ankle-brachial index (ABI), carotid artery intima-media thickness (IMT), and electrocardiogram (ECG). Each measure was scored as 0 (no abnormalities), 1 (minor abnormalities), or 2 (major abnormalities) based on clinically meaningful cutoffs for normal, intermediate, and high values. ABI was obtained as the ratio of ankle systolic blood pressure (BP) to brachial systolic BP, measured by trained technicians following a standard protocol. ${ }^{17} \mathrm{ABI}$ ratio was classified as greater than 1.0 and less than or equal to 1.40 (low risk), $0.90-1.00$ or $>1.40$ (intermediate risk), and $<0.90$ (high risk). Validated ultrasound measurements ${ }^{18}$ were administered using standard scanning and reading protocols across four field centers. ${ }^{19}$ Mean IMT (averaged over left and right sides at each of the bifurcation, internal carotid, or common carotid) was classified as $<0.6 \mathrm{~mm}$ (low risk), $0.6-1.0 \mathrm{~mm}$ (intermediate risk), and $>1.0 \mathrm{~mm}$ (high risk) based on previous original reports in the ARIC Study. ${ }^{20}$ The ECGs were coded using Minnesota Code at the University of Minnesota. ${ }^{21}$ ECG findings were classified based on standardized criteria in the ARIC Study and categorized as no, minor, or major abnormalities (Table S1). ${ }^{22}$ The SVD burden was constructed as the sum of three SVD measures with a score ranging from 0 (lowest burden) to 6 (highest burden). ${ }^{6,23}$

\section{Other Variables}

The participants self-reported age (in years), sex, race, and years of education. Race was categorized as black and white; education was classified as less than high school, high school or equivalent, and more than high school. We created a categorical variable indicating birth cohort (1921-1925, 1926-1930, 19311935, 1936-1940, and 1941-1945). Smoking history (current, previous, and never) and leisure-time physical activity, measured by the Baecke questionnaire (score: 1-4.5), were assessed in a standard interview. Body mass index (BMI) was calculated as body weight $(\mathrm{kg})$ divided by height $(\mathrm{m})$ squared and was 
categorized as underweight $\left(<18.5 \mathrm{~kg} / \mathrm{m}^{2}\right)$, normal $(18.5-<$ $\left.25.0 \mathrm{~kg} / \mathrm{m}^{2}\right)$, overweight $\left(25.0-<30.0 \mathrm{~kg} / \mathrm{m}^{2}\right)$, and obese $\left(\geq 30.0 \mathrm{~kg} / \mathrm{m}^{2}\right)$; underweight and normal categories were collapsed due to small cell size in the underweight category. Presence of diabetes mellitus was identified as fasting serum glucose $\geq 126 \mathrm{mg} / \mathrm{dL}$, non-fasting serum glucose $\geq 200 \mathrm{mg} / \mathrm{dL}$, selfreported use of hypoglycemic medications within the previous two weeks, or self-reported physician diagnosis of diabetes. Systolic and diastolic blood pressure was measured using a standard protocol; anti-hypertensive medication use (yes or no) was self-reported.

Hospitalized CVD events-myocardial infarction, heart failure, and stroke - that occurred during the follow-up period were identified through the ongoing surveillance of medical records and thorough participants' self-report of prior hospitalizations provided using an annual telephone follow-up interview. All CVD events were adjudicated by a panel of clinical investigators as previously described. ${ }^{24-26} \mathrm{~A}$ composite variable was created to indicate the presence of any of three CVD events.

\section{Statistical Analysis}

We described the baseline characteristics by categories of the SVD burden (score: 0, 1, 2, and 3-6) using means and standard deviations for continuous variables and counts and proportions for categorical variables. Choice of cut-points for the SVD burden was determined by the sample distribution of the combined population with and without CVD. Analyses of variance and $\chi^{2}$ test were used to compare continuous and categorical variables, respectively.

We calculated the incidence rates of premature mortality across four SVD categories among the entire sample as well as those with and without prevalent clinical CVD at baseline. Subsequently, we used the Cox proportional-hazards model to determine the adjusted association between SVD burden and premature mortality among persons who were free of clinical CVD at baseline. The SVD burden was modeled as a 4-level categorical variable (score: $0,1,2$, and 3-6), with the lowest burden being the reference (score: 0 ). Model 1 adjusted for a combined race-study center variable, birth cohort, sex, and prevalent CVD (yes or no). Model 2 additionally adjusted for education, smoking status, leisure-time physical activity, BMI, diabetes mellitus, systolic and diastolic blood pressure, and anti-hypertensive medication use. We used Harrell's cstatistic to compare the discrimination between models with and without SVD. Because age at death may be related to birth year, we examined whether the association between SVD burden and premature mortality differed across birth cohorts using a likelihood ratio test comparing two nested models (one model with an interaction term between birth cohort and SVD burden). To compare the effect of SVD burden and clinical CVD on premature mortality, we tested the difference in the point estimates of the highest SVD burden (score: 3-6 vs. score 0 ) and prevalent CVD using the Wald test in models where both persons with and without prevalent CVD at baseline were included.

We conducted several supplementary analyses. First, we included three components of SVD individually in the models to evaluate their respective associations with premature mortality among persons initially free of CVD. Second, we examined the association between SVD burden and premature mortality excluding deaths that occurred in the first two years of follow-up as a sensitivity analysis.

All tests were two-sided with a significance level of $P<0.05$. Analyses were performed using Stata version 15.0 (StataCorp IC, College Station, TX).

\section{RESULTS}

\section{Baseline Characteristics}

On average, persons with a higher SVD burden were older, more likely to be black, had less education and higher BMI, and were more likely to smoke than those with a lower SVD burden (Table 1). A higher SVD burden was also associated with higher prevalence of diabetes, CVD, and antihypertensive medication use as well as higher levels of systolic and diastolic BP. The SVD burden score was skewed to low values, with a median value of 1 and a mean value of 1.5 (Fig. 1). A total of 1868 (13.5\%) scored 0, $6346(45.7 \%)$ scored 1, $3788(27.3 \%)$ scored 2, and $1874(13.5 \%)$ scored $3-6$. For ABI, $80.8 \%, 15.1 \%$, and $4.1 \%$ of the participants scored 0 (no abnormalities), 1 (minor abnormalities), or 2 (major abnormalities), respectively; $22.3 \%, 70.3 \%$, and $7.4 \%$ had a score of 0,1 , and 2 on IMT, respectively; and $68.2 \%$, $26.5 \%$, and $5.3 \%$ scored 0,1 , and 2 on ECG, respectively. A total of 1301 (9.4\%) persons had prevalent CVD at baseline; 625 had heart failure, 578 had myocardial infarction, and 305 had stroke (Table S2). The distribution of the SVD burden score among persons without prevalent CVD was similar to that of the entire sample. Among persons without prevalent CVD at baseline, 1798 (14.3\%) scored 0, 5956 (47.4\%) scored 1, $3406(27.1 \%)$ scored 2, and 1415 (11.2\%) scored 3-6.

\section{Association Between Subclinical Vascular Disease Burden and Premature Mortality}

Over an average of 54.3 years of follow-up (birth date was the time origin), 2010 (9.8\%) premature deaths occurred. Unadjusted incidence rate of premature death was 1.8, 2.2, 2.8, and 4.7 per 1000 person-years (PYs) for persons with an SVD score of 0 (lowest burden), 1, 2, and 3-6 (highest burden), respectively (Table 2 ). For persons without clinically diagnosed CVD at baseline, the unadjusted incidence rate of premature death ranged from 1.7 per 1000 PYs among those with the lowest SVD burden to 3.8 per 1000 PYs among those with the highest SVD burden (Fig. 2). In the population with prevalent CVD, the SVD burden was also able to stratify the incidence rate of premature death among persons with 
Table 1 Baseline Characteristics by Subclinical Vascular Disease Burden; $N=13,876$

\begin{tabular}{|c|c|c|c|c|c|}
\hline & \multicolumn{4}{|c|}{ Subclinical vascular disease burden } & \multirow[b]{2}{*}{$P^{\mathbf{b}}$} \\
\hline & 0 (lowest burden) & 1 & 2 & 3-6 (highest burden) ${ }^{a}$ & \\
\hline & $n=1868$ & $n=6346$ & $n=\mathbf{3 7 8 8}$ & $n=1874$ & \\
\hline \multicolumn{5}{|l|}{ Study center, $n(\%)$} & $<.001$ \\
\hline Forsyth County & $538(28.8)$ & $1629(25.7)$ & $943(24.9)$ & $401(21.4)$ & \\
\hline Jackson & $357(19.1)$ & $1380(21.7)$ & $927(24.5)$ & $553(29.5)$ & \\
\hline Minneapolis & $519(27.8)$ & $1712(27.0)$ & 907 (23.9) & $408(21.8)$ & \\
\hline Washington County & $454(24.3)$ & $1628(25.6)$ & $1011(26.7)$ & $512(27.3)$ & \\
\hline Age, years, mean $\pm \mathrm{SD}$ & $51.5 \pm 5.1$ & $54.0 \pm 5.7$ & $55.0 \pm 5.7$ & $56.6 \pm 5.5$ & $<.001$ \\
\hline Male, $n(\%)$ & $509(27.3)$ & $3020(47.6)$ & $1826(48.2)$ & $887(47.3)$ & $<.001$ \\
\hline Black, $n(\%)$ & $391(20.9)$ & $1539(24.2)$ & 1045 (27.6) & $621(33.1)$ & $<.001$ \\
\hline \multicolumn{5}{|l|}{ Education, $n(\%)$} & $<.001$ \\
\hline$<$ High school & $266(14.2)$ & $1381(21.8)$ & $989(26.2)$ & $652(34.8)$ & \\
\hline$=$ High school & $657(35.2)$ & $2056(32.4)$ & $1238(32.8)$ & $568(30.3)$ & \\
\hline > High school & $945(50.6)$ & $2903(45.8)$ & $1552(41.1)$ & $653(34.9)$ & \\
\hline \multicolumn{5}{|l|}{ BMI, $\mathrm{kg} / \mathrm{m}^{2}, n(\%)$} & $<.001$ \\
\hline$<25$ & $876(46.9)$ & $2094(33.0)$ & 1196 (31.6) & $556(29.7)$ & \\
\hline $25-<30$ & $676(36.2)$ & $2632(41.5)$ & 1505 (39.7) & $730(39.0)$ & \\
\hline$\geq 30$ & $316(16.9)$ & $1623(25.6)$ & $1087(28.7)$ & $588(31.4)$ & \\
\hline \multicolumn{5}{|l|}{ Smoking, $n(\%)$} & $<.001$ \\
\hline Never & $948(50.8)$ & $2778(43.8)$ & $1438(38.0)$ & $603(32.2)$ & \\
\hline Previous & $504(27.0)$ & $2080(32.8)$ & $1267(33.5)$ & $631(33.7)$ & \\
\hline Current & $413(22.1)$ & $1488(23.5)$ & $1081(28.6)$ & $638(34.1)$ & \\
\hline Physical activity ${ }^{\mathrm{c}}$, mean $\pm \mathrm{SD}$ & $2.4 \pm 0.6$ & $2.4 \pm 0.6$ & $2.3 \pm 0.6$ & $2.3 \pm 0.6$ & .005 \\
\hline Diabetes $^{\mathrm{d}}, n(\%)$ & $87(4.7)$ & $627(10.0)$ & $498(13.2)$ & $351(18.9)$ & $<.001$ \\
\hline BP lowering medication, $n(\%)$ & $254(13.7)$ & $1378(21.8)$ & $1050(27.8)$ & $750(40.4)$ & $<.001$ \\
\hline $\mathrm{SBP}, \mathrm{mmHg}$, mean $\pm \mathrm{SD}$ & $113.7 \pm 15.4$ & $120.1 \pm 17.2$ & $122.8 \pm 19.0$ & $128.1 \pm 22.3$ & $<.001$ \\
\hline $\mathrm{DBP}, \mathrm{mmHg}$, mean $\pm \mathrm{SD}$ & $70.9 \pm 10.3$ & $73.7 \pm 10.6$ & $74.1 \pm 11.6$ & $74.5 \pm 12.9$ & $<.001$ \\
\hline Prevalent $\mathrm{CVD}^{\mathrm{e}}, n(\%)$ & $70(3.8)$ & $390(6.1)$ & $382(10.1)$ & $459(24.5)$ & $<.001$ \\
\hline
\end{tabular}

Abbreviations: SD, standard deviation; BMI, body mass index; SBP, systolic blood pressure; DBP, diastolic blood pressure; CVD, cardiovascular disease

${ }^{a} 1,368,376,108$, and 22 scored 3, 4, 5, and 6 on the subclinical vascular disease burden

${ }^{b} P$ values were calculated from chi-square tests or analysis of variance

${ }^{c}$ Physical activity was assessed by the Baecke questionnaire (range: 1-4.5)

${ }^{d}$ Diabetes was identified as fasting glucose level $\geq 126 \mathrm{mg} / \mathrm{dL}$, non-fasting serum glucose $\geq 200 \mathrm{mg} / \mathrm{dL}$, self-reported use of hypoglycemic medications, or self-reported physician diagnosis within the previous two weeks

${ }^{e}$ Including myocardial infarction, heart failure, and stroke

prevalent CVD at baseline; the rate ranged from 3.2 per 1000 PYs among those with the lowest SVD burden to 7.4 per 1000 PYs among those with the highest SVD burden.

Among persons initially free of clinically diagnosed CVD, the hazards of premature death among those with the highest SVD burden (score: 3-6) were approximately two times $(\mathrm{HR}=2.05,95 \% \mathrm{CI}: 1.68,2.50)$ as high as the lowest-risk group after adjustment of demographics (score: 0; Table 2). The association between high SVD burden and premature death slightly attenuated but persisted after adjustment for additional confounders (HR $=1.47,95 \%$ CI: $1.19,1.79)$. Results did not change substantially in the sensitivity analysis in which persons who died within the first two years were excluded (Table S3). Harrell's $c$-statistics for the full models with and without people with clinically diagnosed CVD were 0.74 and 0.65 , respectively, showing moderate discrimination for separating persons who had and did not have premature mortality. There was little evidence of effect modification according to birth cohort ( $P$ for model comparison using likelihood ratio test: 0.28 )

In the model where persons with and without clinically diagnosed CVD at baseline were both included, we observed that the association of high SVD burden (score: 3-6 vs. 0) with premature death was equivalent to that between prevalent CVD and premature death $(\mathrm{HR}=1.49$ vs. $1.68 ; P$ for comparison $=0.32$; Table 3 ).

We also evaluated the respective associations of the three components (ABI, IMT, and ECG) of the SVD burden index with premature mortality. The results showed that all three components contributed similarly and significantly to premature mortality, suggesting that premature mortality is not driven solely by just one or two of the components (Table S4).

\section{DISCUSSION}

To our knowledge, this is the first large-scale study of SVD and premature mortality (death before 70 years of age) among middle-aged adults in the USA. Four findings are worth mentioning. First, this study illustrated the wide range of SVD among middle-aged adults with or without clinical CVD. Second, we found that SVD burden-assessed by ABI, IMT, and ECG - was associated with premature mortality, independent of demographics, lifestyles, and clinical CVD. Third, our findings showed that the association between high SVD burden and premature mortality was not hugely different from that observed for clinical CVD. Taken together, these results suggest that measures of SVD are important for 

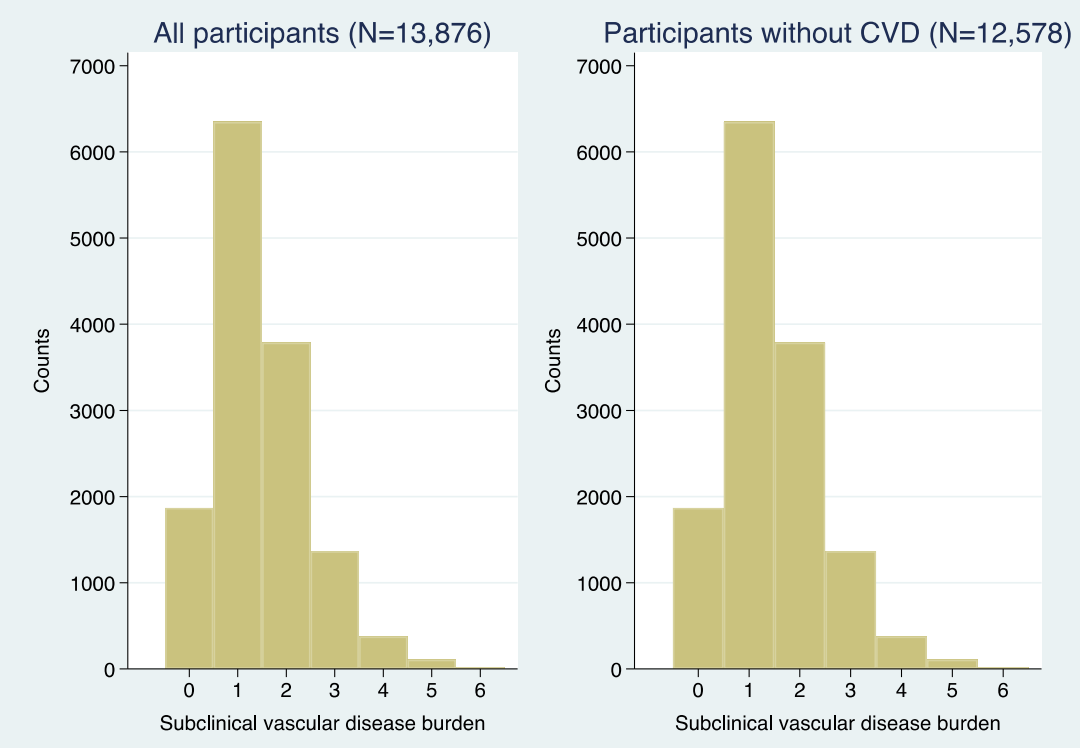

Figure 1 Distribution of the subclinical vascular burden among all persons $(n=13,876)$ and those without prevalent cardiovascular disease (CVD) at baseline $(n=12,578)$.

capturing the full spectrum of cardiovascular comorbidity in midlife.

Prior investigations have shown a strong association between SVD burden and clinical CVD events, mortality, and survival among older adults. Using data from the Cardiovascular Health Study, Inzitari et al. ${ }^{23}$ showed that a higher SVD burden was associated with increased risk of CVD and mortality among 3252 persons aged at least 65 years. Using data from the same cohort, Odden et al. ${ }^{6}$ found that a lower burden of SVD was associated with longer survival among 2082 persons aged 70-75 years. However, existing research on SVD has focused on older populations, with relatively little

Table 2 Association of Subclinical Vascular Disease Burden and Mortality Among Persons Without Clinically Diagnosed Cardiovascular Disease at Baseline; $N=\mathbf{1 2 , 5 7 8}$

\begin{tabular}{|c|c|c|c|c|}
\hline & \multicolumn{2}{|c|}{ Premature death } & \multirow{2}{*}{$\begin{array}{l}\begin{array}{l}\text { Demographic } \\
\text { adjusted }^{\mathrm{a}}\end{array} \\
\begin{array}{l}\text { Hazard ratio (95\% } \\
\text { interval) }\end{array}\end{array}$} & \multirow{2}{*}{$\begin{array}{l}\begin{array}{l}\text { Fully } \\
\text { adjusted }^{\mathbf{b}}\end{array} \\
\text { confidence }\end{array}$} \\
\hline & Deaths & $\begin{array}{l}\text { Rates per } \\
1000 \text { PYs }\end{array}$ & & \\
\hline \multicolumn{5}{|c|}{ SVD burden } \\
\hline & 160 & $\begin{array}{l}1.7(1.5, \\
2.0)\end{array}$ & Ref. & Ref. \\
\hline 1 & 675 & $\begin{array}{l}2.1(1.9, \\
2.3)\end{array}$ & $1.11(0.92,1.31)$ & $\begin{array}{l}0.99(0.83, \\
1.19)\end{array}$ \\
\hline 2 & 470 & $\begin{array}{l}2.5(2.3, \\
2.8)\end{array}$ & $1.32(1.10,1.58)$ & $\begin{array}{l}1.08(0.89, \\
1.30)\end{array}$ \\
\hline $\begin{array}{c}\text { 3-6 } \\
\text { (highest) }\end{array}$ & 303 & $\begin{array}{l}3.8(3.4, \\
4.3)\end{array}$ & $2.05(1.68,2.50)$ & $\begin{array}{l}1.47(1.19, \\
1.79)\end{array}$ \\
\hline
\end{tabular}

Abbreviations: PY, person-year; SVD, subclinical vascular disease, $C V D$, cardiovascular disease

${ }^{a}$ Demographic adjusted model adjusted for sex, race-center, and birth cohort

${ }^{b}$ Fully adjusted model adjusted for sex, race-center, birth cohort, body mass index, smoking status, physical activity, diabetes, anti-hypertensive medication, and blood pressure attention on middle adulthood - a potentially critical period for accumulating risk for CVD events and death.

Previous longitudinal and cross-sectional investigations suggest that midlife SVD burden results from long-term exposure to a wide range of modifiable socio-demographic, behavioral, nutritional, physiological, and environmental risk factors - both traditional and nontraditional. ${ }^{27-32}$ Thus, our study fills a much-needed research gap by depicting the wide range of SVD burden among middle-aged adults and demonstrating its relationship with premature mortality.

Clinical CVD has a prevalence of less than 10\% among midlife adults, ${ }^{15}$ and is therefore unlikely to fully account for vascular burden in this population. The present study demonstrated that middle-aged adults with and without clinical CVD had hugely different levels of SVD burden; these levels were corresponding with different risks of premature death. These findings suggest that measures of SVD are essential for capturing the full spectrum of cardiovascular comorbidity and could provide additional risk stratification of death beyond clinical CVD in midlife. Another intriguing finding is that the association between high SVD burden and premature mortality was not hugely different from that observed for clinical CVD, underscoring the importance of subclinical measures in risk assessment and management among middle-aged adults.

Our study has many strengths, including its prospective design, comprehensive set of measurements of potential confounders, long follow-up period, large population-based sample, heterogeneity in demographic composition of the study sample, and adjudicated CVD events. Despite these strengths, we acknowledge several limitations. First, although we showed the wide range of SVD among middle-aged adults with or without clinical CVD, we did not explore potential mechanisms through which SVD may influence premature 


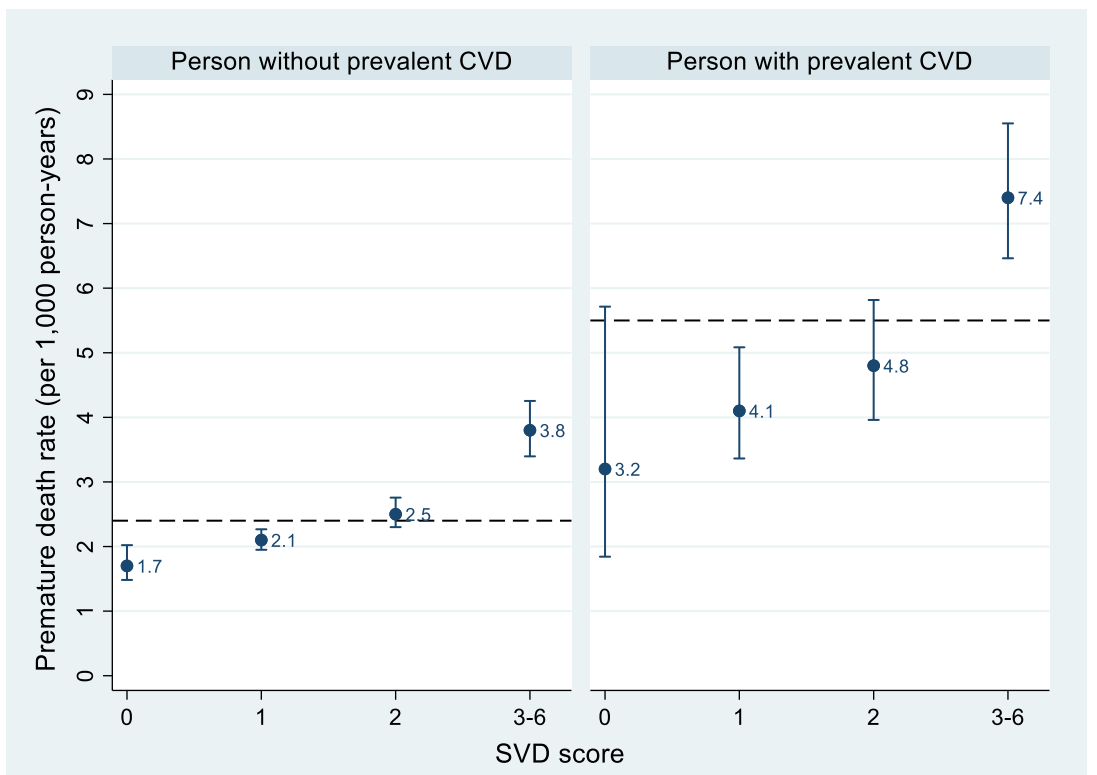

Figure 2 Incidence rates with $95 \%$ confidence interval of premature mortality (died before 70 years of age) across categories of subclinical vascular disease (SVD) burden among persons with and without clinically diagnosed cardiovascular disease (CVD) at baseline. The black dashed line represents estimate of marginal probability of premature death within each subgroup.

mortality. Future research needs to examine more intermediate health measures - for example, frailty and functional status - to elucidate the pathways. Second, we demonstrated the strong association of SVD burden with premature mortality and the ability of the SVD burden to stratify premature death beyond clinical CVD among middle-aged adults. However, the present investigation is mainly an association study, which did not adequately address the question of whether measures of SVD could improve prediction for premature mortality. We found that inclusion of SVD measures could improve the discrimination ability for predicting premature death. Future research using predictive modeling techniques ${ }^{33}$ is needed to more comprehensively investigate the added prognostic value of SVD measures. Third, all three measures of SVD were measured only at baseline; these measures may vary over time, potentially leading to misclassification. Fourth, about $10 \%$ of the ARIC participants were not included in the present study due to missing data in SVD measures. These persons were unhealthier than those included (data not shown), which might lead to underestimate the association between SVD burden and premature mortality. Fifth, we considered an ABI of 0.90-1.00 or $>1.40$ as an intermediate risk in the present study. However, an ABI > 1.40 might represent a different pathology such as medial arterial calcification. We reclassified the ABI ratio into a 4-level categorical variable with $\mathrm{ABI}>1.40$ as an independent group. We examined its association with premature mortality and found the difference between ABI of $0.9-1.0$ and ABI $>1.4$ was not statistically significant (data not shown). Lastly, we did not differentiate deaths from CVD and those from non-CVD causes because our main purpose was to demonstrate the importance of SVD measures in stratifying the risk of premature mortality. Future inquiry could identify the association between SVD and cause-specific mortality.

In summary, middle-aged adults with a higher burden of SVD had an increased hazard of premature mortality. This study demonstrated that the SVD index could be beneficial for more fully capturing vascular burden. Taken together, our findings suggest the importance of evaluation of SVD among middle-aged adults both with and without clinical CVD. The

Table 3 Association of Subclinical Vascular Disease Burden and Prevalent Cardiovascular Disease with Premature Mortality. $N=$ 13,876

\begin{tabular}{|c|c|c|c|}
\hline \multirow[b]{2}{*}{$\begin{array}{l}\text { Premature } \\
\text { mortality }\end{array}$} & \multirow[b]{2}{*}{$\begin{array}{l}\text { Rates per } 1000 \\
\text { PYs }\end{array}$} & Model 1 $^{\mathrm{a}}$ & Model $2^{b}$ \\
\hline & & $\begin{array}{l}\text { Hazard rat } \\
\text { confidence }\end{array}$ & $\begin{array}{l}5 \% \\
\text { rval) }\end{array}$ \\
\hline \multicolumn{4}{|l|}{ SVD burden } \\
\hline 0 (lowest) & $1.8(1.5,2.1)$ & Ref. & Ref. \\
\hline 1 & $2.2(2.1,2.4)$ & $\begin{array}{l}1.09(0.92, \\
1.28)\end{array}$ & $\begin{array}{l}0.99(0.83, \\
1.17)\end{array}$ \\
\hline 2 & $2.8(2.5,3.0)$ & $\begin{array}{l}1.30(1.09, \\
1.55)\end{array}$ & $\begin{array}{l}1.10(0.92, \\
1.31)\end{array}$ \\
\hline 3-6 (highest) & $4.7(4.3,5.1)$ & $\begin{array}{l}1.97(1.64, \\
2.36)\end{array}$ & $\begin{array}{l}1.49 \text { (1.23, } \\
1.79)\end{array}$ \\
\hline \multicolumn{4}{|l|}{ Prevalent $\mathrm{CVD}^{\mathrm{c}}$} \\
\hline No & $2.4(2.3,2.5)$ & Ref. & Ref. \\
\hline Yes & $5.5(5.0,6.0)$ & $\begin{array}{l}1.91(1.70, \\
2.14)\end{array}$ & $\begin{array}{l}1.68(1.48, \\
1.90)\end{array}$ \\
\hline
\end{tabular}

Abbreviations: PYs, person-years; SVD, subclinical vascular disease; $C V D$, cardiovascular disease

${ }^{a}$ Model 1 adjusted for sex, race-center, and birth cohort

${ }^{b}$ Model 2 adjusted for sex, race-center, birth cohort, body mass index, smoking status, physical activity, diabetes, anti-hypertensive medication, and systolic and diastolic blood pressure

${ }^{c}$ Including myocardial infarction, heart failure, and stroke $P$ value for comparison between effects of high SVD burden (score: 3-6) and prevalent CVD on premature death was 0.82 in model 1 and 0.32 in model 2 
SVD index relies on only three measures (ABI, IMT, and ECG), which are available in many epidemiological studies and can be administered noninvasively and relatively inexpensively in clinical settings. It is important to take SVD into account when designing interventions for reducing premature mortality.

Acknowledgments: The Atherosclerosis Risk in Communities Study is carried out as a collaborative study supported by National Heart, Lung, and Blood Institute contracts (HHSN268201100005C, HHSN $268201100006 \mathrm{C}$, HHSN2682011000O7C, HHSN $268201100008 \mathrm{C}$, HHSN268201100009C, HHSN $268201100010 C$, HHSN268201100011C, and HHSN268201100012C) with the ARIC carotid MRI examination funded by U01HL075572-01. The authors thank the staff and participants of the ARIC Study for their important contributions. Dr. Chenkai Wu is supported by the Suzhou Municipal Science and Technology Bureau (SS2019069).

Corresponding Author: Chenkai Wu, Ph.D., M.P.H., M.S.; Global Health Research Center, Duke Kunshan University, Kunshan 215316, Jiangsu, China (e-mail: chenkai.wu@dukekunshan.edu.cn).

\section{Compliance with Ethical Standards:}

Conflict of Interest: Dr. Chenkai Wu provides paid consultant services to HealthKeeperS, a health data analytics company in China. Dr. Michelle Odden provides paid consultant services to Cricket Health, Inc., a kidney care company in the USA.

\section{REFERENCES}

1. Norheim, O. F. et al. Avoiding $40 \%$ of the Premature Deaths in Each Country, 2010-30: Review of National Mortality Trends to Help Quantify the UN Sustainable Development Goal for Health. Lancet 385, 239-252 (2015).

2. Bennett, J. E. et al. NCD Countdown 2030: worldwide trends in noncommunicable disease mortality and progress towards Sustainable Development Goal target 3.4. Lancet, 392, 1072-1088 (2018).

3. “Cardiovascular Diseases." February 28, 2020. https://www.who.int/ westernpacific/health-topics/cardiovascular-diseases.

4. “Heart Disease and Stroke," April 1, 2019. https://www.cdc.gov/ chronicdisease/resources/publications/factsheets/heart-diseasestroke.htm.

5. Newman, A. B. et al. Associations of subclinical cardiovascular disease with frailty. J Gerontol A Biol Sci Med Sci. 56, M158-166 (2001).

6. Odden, M. C. et al. Subclinical vascular disease burden and longer survival. J Am Geriatr Soc. 62, 1692-1698, doi:https://doi.org/10.1111/ jgs. 13018 (2014).

7. Inzitari, M. et al. Subclinical vascular disease burden and risk for death and cardiovascular events in older community dwellers. J Gerontol Ser A: Biomed Sci Med Sci. 66, 986-993 (2011).

8. Newman, A. B. et al. Ankle-arm index as a predictor of cardiovascular disease and mortality in the Cardiovascular Health Study. Arterioscler Thromb Vasc Biol. 19, 538-545 (1999).

9. Newman, A. B. et al. "Successful aging": effect of subclinical cardiovascular disease. Arch Intern Med. 163, 2315-2322, doi:https://doi.org/10. 1001/archinte.163.19.2315 (2003).

10. Newman, A. B., Boudreau, R. M., Naydeck, B. L., Fried, L. F. \& Harris, T. B. A physiologic index of comorbidity: relationship to mortality and disability. J Gerontol Ser A: Biol Sci Med Sci 63, 603-609 (2008).

11. Wu, C., Smit, E., Sanders, J. L., Newman, A. B. \& Odden, M. C. A Modified Healthy Aging Index and Its Association with Mortality: The National Health and Nutrition Examination Survey, 1999-2002. J Gerontol A Biol Sci Med Sci. 72, 1437-1444, doi:https://doi.org/10. 1093/gerona/glw334 (2017).
12. Chaves, $\mathbf{P}$. H. et al. Subclinical cardiovascular disease in older adults: insights from the Cardiovascular Health Study. Am J Geriatr Cardiol. 13, 137-151 (2004).

13. Kuller, L. H. et al. Subclinical disease as an independent risk factor for cardiovascular disease. Circulation 92, 720-726 (1995).

14. Kuller, L. H. et al. Diabetes mellitus: subclinical cardiovascular disease and risk of incident cardiovascular disease and all-cause mortality. Arterioscler Thromb Vasc Biol. 20, 823-829 (2000).

15. Benjamin, E. J. et al. Heart disease and stroke statistics-2017 update: a report from the American Heart Association. Circulation 135, e146e603 (2017)

16. The Atherosclerosis Risk in Communities (ARIC) Study: design and objectives. The ARIC investigators. Am J Epidemiol. 1989;129:687-702.

17. Mundt, K. A., Chambless, L. E., Burnham, C. B. \& Heiss, G. Measuring ankle systolic blood pressure: validation of the Dinamap $1846 \mathrm{SX}$. Angiology 43, 555-566, doi:https://doi.org/10.1177/ 000331979204300703 (1992).

18. Pignoli, P., Tremoli, E., Poli, A., Oreste, P. \& Paoletti, R. Intimal plus medial thickness of the arterial wall: a direct measurement with ultrasound imaging. Circulation 74, 1399-1406 (1986).

19. High-resolution B-mode ultrasound reading methods in the Atherosclerosis Risk in Communities (ARIC) cohort. The ARIC Study Group. J Neuroimaging. 1991;1:168-172.

20. Chambless, L. E. et al. Association of coronary heart disease incidence with carotid arterial wall thickness and major risk factors: the Atherosclerosis Risk in Communities (ARIC) Study, 1987-1993. Am J Epidemiol. 146, 483-494 (1997)

21. Prineas RJ, Crow R, Blackburn HW. The Minnesota Code Manual of Electrocardiographic Findings: Standards and Procedures for Measurement and Classification. J. Wright; Boston, MA: 1982.

22. Vitelli, L. L. et al. Electrocardiographic findings in a healthy biracial population. Atherosclerosis Risk in Communities (ARIC) Study Investigators. Am J Cardiol. 81, 453-459 (1998).

23. Inzitari, M. et al. Subclinical vascular disease burden and risk for death and cardiovascular events in older community dwellers. J Gerontol A Biol Sci Med Sci. 66, 986-993, doi:https://doi.org/10.1093/gerona/glr069 (2011).

24. Rosamond, w.D. et al. Trends in the incidence of myocardial infarction and in mortality due to coronary heart disease, 1987 to 1994 . N Engl J Med. 339, 861-867 (1998)

25. Rosamond, w.D. et al. Classification of heart failure in the Atherosclerosis Risk in Communities (ARIC) study: a comparison of diagnostic criteria. Circ Heart Fail. 5, 152-159 (2012).

26. Jones, S.A. et al. Validity of hospital discharge diagnosis codes for stroke: the Atherosclerosis Risk in Communities Study. Stroke 45, 3219-3225 (2014).

27. Carson, A. P. et al. Cumulative socioeconomic status across the life course and subclinical atherosclerosis. Ann Epidemiol. 17, 296-303, doi:https://doi.org/10.1016/j.annepidem.2006.07.009 (2007).

28. Nieto, F. J., Diez-Roux, A., Szklo, M., Comstock, G. W. \& Sharrett, A. R. Short- and long-term prediction of clinical and subclinical atherosclerosis by traditional risk factors. J Clin Epidemiol. 52, 559-567 (1999).

29. Diez Roux, A. V. et al. Long-term exposure to ambient particulate matter and prevalence of subclinical atherosclerosis in the Multi-Ethnic Study of Atherosclerosis. Am J Epidemiol. 167, 667-675, doi:https://doi.org/10. 1093/aje/kwm359 (2008).

30. Psaty, B. M. et al. Association between levels of blood pressure and measures of subclinical disease multi-ethnic study of atherosclerosis. Am J Hypertens. 19, 1110-1117, doi:https://doi.org/10.1016/j.amjhyper. 2006.04.002 (2006).

31. Allison, M. A. et al. Prevalence of and risk factors for subclinical cardiovascular disease in selected US Hispanic ethnic groups: the Multi-Ethnic Study of Atherosclerosis. Am J Epidemiol. 167, 962-969, doi:https://doi.org/10.1093/aje/kwm402 (2008).

32. Lutsey, P. L. et al. Associations of acculturation and socioeconomic status with subclinical cardiovascular disease in the multi-ethnic study of atherosclerosis. Am J Public Health. 98, 1963-1970, doi:https://doi. org/10.2105/AJPH.2007.123844 (2008).

33. Steyerberg, E. W. et al. Assessing the performance of prediction models: a framework for traditional and novel measures. Epidemiology 21, 128138, doi:https://doi.org/10.1097/EDE.0b013e3181c30fb2 (2010).

Publisher's Note: Springer Nature remains neutral with regard to jurisdictional claims in published maps and institutional affiliations. 